\title{
PERIOPERATIVE PAIN MANAGEMENT IN PRIMARY BONE TUMORS
}

doi: 10.2478/rojost-2018-0019

\author{
A. Bratu' ${ }^{1,2}$, Z. Panti ${ }^{2,3}$, A. Comanelea ${ }^{1}$, R. Ene ${ }^{2,3}$, C. Cîrstoiu' ${ }^{2,3}$ \\ ${ }^{1}$ Anesthesiology and Intensive Care Department, University Emergency Hospital, Bucharest, Romania \\ ${ }^{2}$ Orthopedics and Traumatology Department, University Emergency Hospital, Bucharest, Romania \\ 3"Carol Davila" University of Medicine and Pharmacy, Bucharest, Romania
}

Introduction. The increasing number of tumor prosthesis in the last decade shows the advance in musculoskeletal oncology. Limb sparing surgery nowadays has to be the focus in surgery, maintaining the patient's quality of life.

Prognosis depends on the histological type of tumor, size, and local extension. Pain is present in almost any cases of bone tumors and can vary in intensity and character. Being the leading symptom is strongly correlated to the quality of life.

The purpose of this study was to evaluate pain in patients with primary bone sarcomas before and after surgery.

Material and methods. 11 patients were involved in this study over a period of 4 years (2014-2017) from the Orthopedics and Traumatology Department in the University Emergency Hospital in Bucharest. Tumor resection and reconstruction with modular prosthesis was performed in 4 cases, and tumor resection was necessary in 3 cases and amputation in 4 cases. Pain was evaluated before and after surgery using the Visual Analog Scale (VAS). Early postoperative pain control was achieved with epidural catheter, followed by opioid therapy, NSAIDs and Paracetamol in the early stages of mobilization.

Results. Surface sarcomas and tumors close to the periosteum, or periosteal involvement has shown a localized and increased pain. Multimodal-analgesia was used for pain management. Within the first 48 hours, analgesia was performed with an epidural catheter by continuous infusion of ropivacaine $0.2 \%$ and fentanyl $2 \mathrm{mcg} / \mathrm{ml}$ at a rate ranging between $3-6 \mathrm{ml} / \mathrm{h}$, obtaining a VAS score between $o$ and 3 .

Conclusions. Perioperative pain management has to be individualized to the localization and local soft tissue involvement of the tumor. In late stages of sarcomas or local recurrence, conventional analgesics can be inefficient. Early diagnosis and surgical removal of these tumors is the most important objective for a good prognosis.

Keywords: pain, bone sarcomas, prognosis 